\title{
Recent Developments in Technology for Horizontally Aligned Growth of Single-Walled Carbon Nanotubes
}

\author{
Hiroki Ago ${ }^{1,2,3, *}$, Carlo M. Orofeo ${ }^{2}$, Naoki Ishigami², \\ Naoki Yoshihara ${ }^{2}$ and Masaharu Tsuji ${ }^{1}$ \\ 'Institute for Materials Chemistry and Engineering, Kyushu University, Fukuoka 816-8580, Japan \\ ${ }^{2}$ Graduate School of Engineering Sciences, Kyushu University, Fukuoka 816-8580, Japan \\ ${ }^{3}$ PRESTO, Japan Science and Technology Agency (JST), Kawaguchi-shi, Saitama 332-0012, Japan
}

(Received April 16, 2009; accepted June 1, 2009)

Key words: single-walled carbon nanotubes, sapphire, silicon, alignment, nanoelectronics

Horizontally aligned growth of single-walled carbon nanotubes (SWNTs) on a substrate is an essential step towards the formation of rational architectures for electronic applications. We present our recent progress in horizontally aligned SWNT growth on a single-crystal sapphire substrate, in which two different alignment mechanisms, lattice-oriented growth and step-templated growth, were observed. Characterization of aligned SWNTs on sapphire suggests the crystal plane dependence of diameter and chiral angle distributions. In addition, our recent achievements in aligned SWNT growth on a surface-modified Si substrate is also demonstrated.

\section{Introduction}

A carbon nanotube, a highly crystalline one-dimensional nanomaterial, shows extraordinary high carrier mobility and current density, as well as excellent mechanical flexibility and chemical stability. These properties are promising for applications in electronics. ${ }^{(1)}$ Single-walled carbon nanotubes (SWNTs) possess a very narrow diameter range of 1-2 $\mathrm{nm}$ and a unique electronic structure with a wide range of band gaps $(0-1 \mathrm{eV})$ that depends on chirality and diameter. Semiconducting SWNTs are potentially applicable to field-effect transistors (FETs) and optoelectronic devices, whereas metallic SWNTs can be applied to transparent metallic electrodes. In addition, the excellent mechanical property of SWNTs provides flexibility in these devices. However, the following two important issues need to be addressed in order to develop SWNT-based devices: (i) integration of a number of SWNTs on a substrate and (ii) controlled growth of SWNTs with a desired electronic structure or chirality.

In spite of a large number of studies on carbon nanotubes, the above issues still remain unresolved and hinder the wide-scale electronic applications using nanotubes. Because these problems are common for various nanoscale materials, it is very important

*Corresponding author: e-mail: ago@cm.kyushu-u.ac.jp 
to study these issues for future development of nanotechnology.

For nanotube integration, the control over both growth direction and position of SWNTs is required. Although vertically aligned SWNT arrays have attracted much interest for a long time in terms of their observed high catalytic activity, horizontally aligned SWNT arrays are becoming more important for electronics because most electronic devices have a lateral geometry and the horizontal alignment enables access to each nanotube by depositing electrodes using lithography techniques. The control over electronic structure or chirality during nanotube growth is more challenging. Recently developed separation techniques, such as density-gradient, polymer wrapping, and filtration methods, have enabled metal-semiconductor or chirality separation. However, these separation methods are only applicable to SWNT dispersion, and dispersed SWNTs suffer from many defects and contamination. Therefore, there is a strong demand for the control of SWNT structure during SWNT growth.

\section{Horizontally Aligned Growth on Single-Crystal Surfaces}

We studied the possibility of "epitaxial SWNT growth" by synthesizing SWNTs on single-crystal substrates to control the nanotube structure. Previously, we discovered the horizontally aligned growth of SWNTs on sapphire substrates $\left(\alpha-\mathrm{Al}_{2} \mathrm{O}_{3}\right)$ with a-plane (1120) and r-plane (1102). ${ }^{(2)}$ Figures 1(a) and 1(b) show scanning electron microscopy (SEM) images of SWNTs grown on the r-plane sapphire and $\mathrm{SiO}_{2} / \mathrm{Si}$ substrates, respectively. The SWNTs were grown by chemical vapor deposition (CVD) of the methane $\left(\mathrm{CH}_{4}\right)$
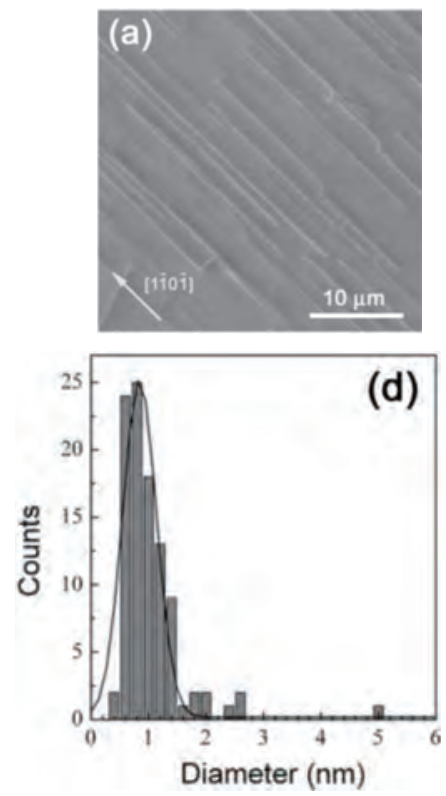
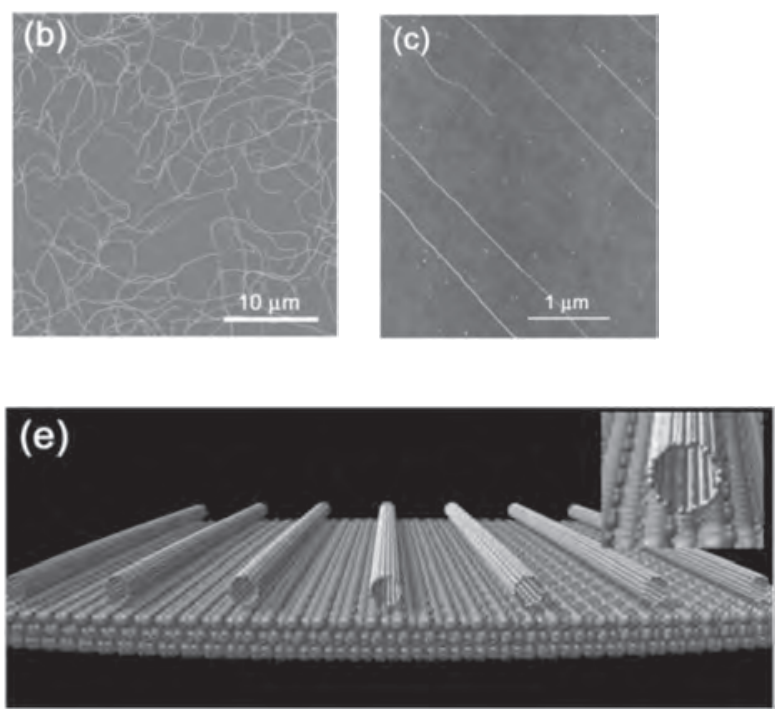

Fig. 1. SEM images of SWNTs grown on sapphire r-plane (a) and $\mathrm{SiO}_{2} / \mathrm{Si}$ (b) substrates. AFM images of aligned SWNTs on r-plane (c) and corresponding height histogram (d). (e) Schematic image of aligned SWNTs on sapphire a-plane. 
source at $900^{\circ} \mathrm{C}$. Most of the SWNTs are aligned parallel to the [1101] direction on the r-plane sapphire, whereas SWNTs on the silicon substrate are randomly oriented. An atomic force microscopy (AFM) image (Fig. 1(c)) and a height histogram (Fig. 1(d)) indicate that the aligned nanotubes are generally isolated and have a mean diameter of 1.0 $\mathrm{nm}$. The polarized Raman measurement confirmed that the aligned nanotubes are mainly single-walled and oriented in a specific crystallographic direction. ${ }^{(3)}$

Our results suggest that the highly ordered crystalline surface of the sapphire substrate plays an important role in SWNT alignment, because the $\mathrm{SiO}_{2}$ layer on the silicon substrate has an amorphous structure. We speculate that an anisotropic van der Waals interaction between an SWNT and the sapphire surface guides the aligned growth. The atomic model is illustrated in Fig. 1(e). Because the growth direction of SWNTs is predetermined by the crystallographic direction of the sapphire substrate, we call this phenomenon "atomic-arrangement programmed growth (AAP growth)." This aligned growth has only been reported for single-crystal sapphire ${ }^{(2,4)}$ and quartz $\left(\mathrm{SiO}_{2}\right),{ }^{(5)}$ mainly because most other single crystals are not stable in a reductive atmosphere at a high CVD temperature.

\section{Control of Growth Position and Unidirectional Growth}

In CVD growth, SWNT position is basically controlled by defining the catalyst using a lithography technique. Through catalyst patterning on sapphire substrates, both growth direction and position of SWNTs become controllable. Generally, SWNTs grown from the patterned catalyst were extended in both directions, as shown in Fig. 2(a).
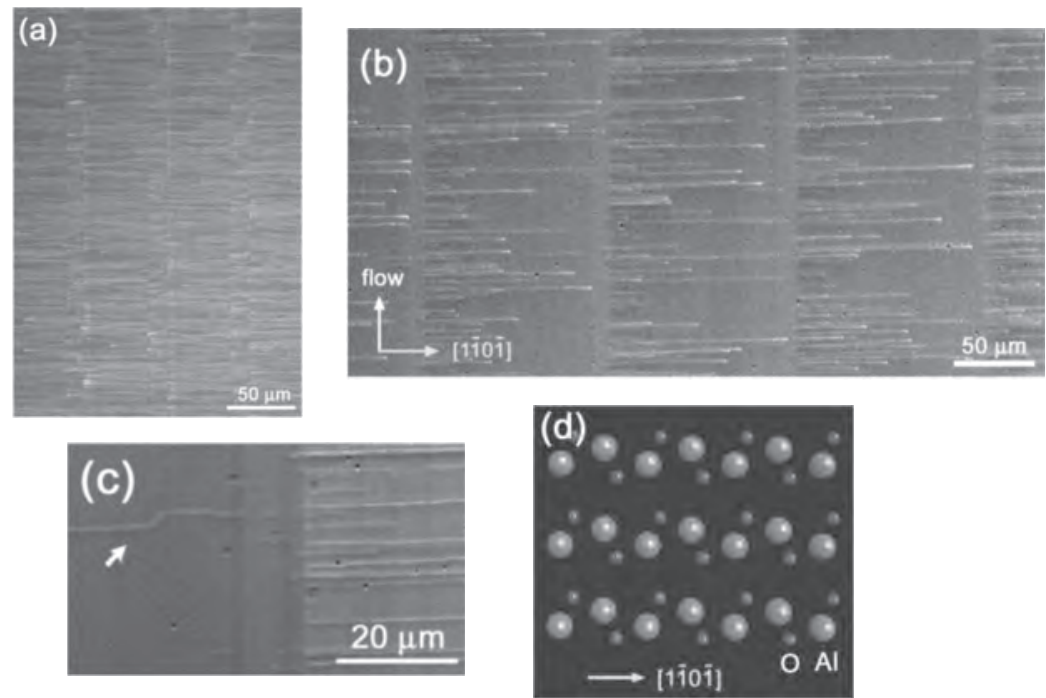

Fig. 2. (a) SEM image of SWNTs grown from patterned catalyst. The SWNTs extend in both directions from the pattern. (b,c) SEM images of unidirectional SWNTs grown on r-plane sapphire. The SWNTs preferentially grew in the [1101] direction. The arrow in (c) indicates the SWNT grown from the left catalyst line. (d) Surface atomic model of sapphire r-plane. 
However, we have recently found that aligned SWNTs grow preferentially in the [1101] direction on r-plane sapphire (Figs. 2(b) and 2(c)). ${ }^{(6)}$ This unidirectional growth was not as pronounced when a sputtered metal film was used as the catalyst. When we used the Co-Mo salt metal as the catalyst, the unidirectional growth was observed at a high yield $(\sim 96 \%)$. We noted that this unidirectional growth did not occur on sapphire a-plane and ST-cut quartz, on which SWNTs grew in both directions from the patterned catalyst. Thus, the unidirectional growth is a unique phenomenon observed only on the sapphire r-plane. Figure 2(d) shows the surface atomic arrangement of the r-plane. Note that the atomic configuration in the [1101] direction differs from that in the opposite direction. On the other hand, the sapphire a-plane has point symmetry so that the atomic configuration is essentially the same for both directions. Therefore, the observed unidirectional growth is attributed to the highly anisotropic surface atomic arrangement of the r-plane. The fact that the nanotube growth direction is determined by the atomic arrangement reflects a strong effect of the substrate surface on SWNTs during their growth.

\section{Alignment Mechanism}

The growth mechanisms of carbon nanotubes can be classified into two growth modes. These are labeled depending on whether the catalyst is located at the base (basegrowth mode) or tip (tip-growth mode) of a nanotube during its growth. The growth mode of aligned SWNTs on sapphire is closely related to the alignment mechanism. In the case of the base-growth mode, nanotube-substrate interaction should play an important role, whereas either catalyst-substrate or nanotube-substrate interaction is important for the tip-growth mode. The growth mode is also helpful for a highly controlled SWNT growth, but it was difficult to distinguish these two modes by AFM and SEM measurements.

We clarified the growth mode by forming a gradation of carbon isotopes in a SWNT along its long axis. Figure 3(a) shows the reaction scheme of this carbon isotopelabeling experiment. After reaching a CVD temperature of $900^{\circ} \mathrm{C},{ }^{13} \mathrm{CH}_{4}$ gas was firstly introduced, and after $1.5 \mathrm{~min},{ }^{12} \mathrm{CH}_{4}$ gas was added and the reaction was allowed to last for another $1 \mathrm{~min}$. For the base-growth SWNT, the initially introduced ${ }^{13} \mathrm{C}$-rich tube is expected to be located at the nanotube tip, and ${ }^{12} \mathrm{C}$ concentration increases towards the catalyst (Fig. 3(b)). The opposite isotope profile is expected for the tip-growth mode, because the initially formed nanotube cap should remain at the catalyst pattern. The Fe catalyst was patterned by electron beam lithography to define the starting position.

Spatial isotope distribution was studied by Raman mapping measurement. The Raman G-band appears usually at $1,592 \mathrm{~cm}^{-1}$ for a ${ }^{12} \mathrm{C}$-labeled SWNT, but it shifts to $1,530 \mathrm{~cm}^{-1}$ when all the carbon atoms are replaced by ${ }^{13} \mathrm{C} .{ }^{(7)}$ This large G-band shift can be used to monitor the isotope distribution in one SWNT. A representative result is shown in Fig. 3(c). ${ }^{(8)}$ A long nanotube with a length of $76 \mu \mathrm{m}$ was clearly observed by Raman mapping. These mapping images show that the tip mainly consists of ${ }^{13} \mathrm{C}$, and the relative ratio of ${ }^{12} \mathrm{C}$ increases towards the body. Other nanotubes also showed similar isotope profiles. These observations reveal the base-growth mode of the present SWNT, which indicates the importance of nanotube-substrate interaction. 


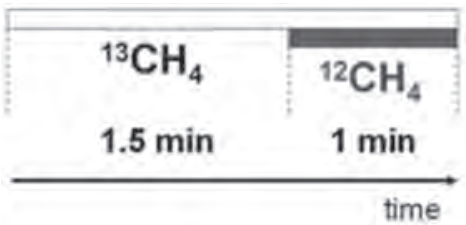

(a)

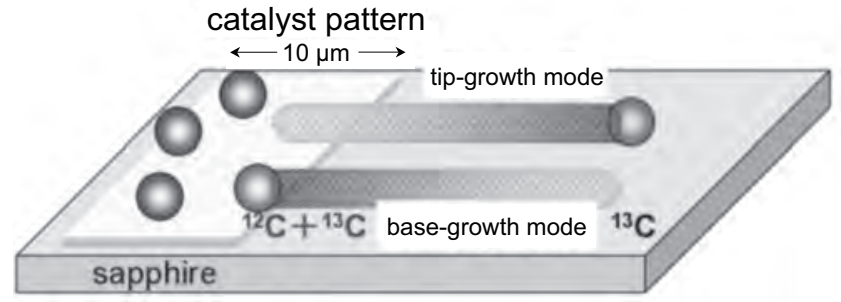

(b)

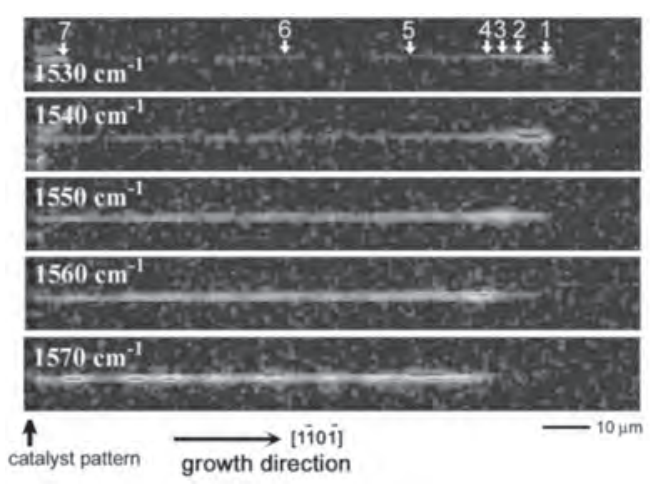

(c)

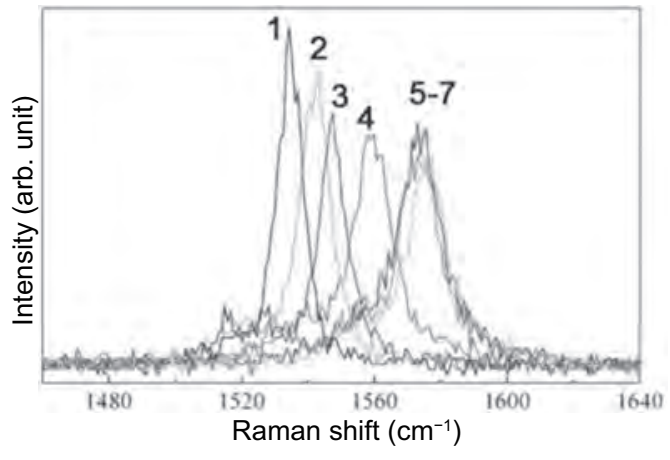

(d)

Fig. 3. Reaction scheme of carbon isotope-labeled CVD experiment (a) and expected isotope distributions in aligned SWNTs for base- and tip-growth modes (b). (c) Spatial Raman G-band intensity of one SWNT measured for different wave numbers. (d) Raman spectra measured for the different positions numbered in (c).

It is still unclear why such long SWNTs were obtained through the interaction of SWNTs with the substrate underneath. However, it seems that this confounds intermolecular force with macroscopic friction force. Because the sapphire surface is atomically smooth, the aligned SWNT is expected not to suffer from strong friction force once it starts to grow, and slides along the very clean sapphire surface.

\section{Characterization of SWNTs Grown on Sapphire}

The observations of the unidirectional growth and the base-growth mode suggest that the aligned SWNT growth is strongly affected by the sapphire surface. It is, therefore, interesting to investigate the aligned SWNTs in terms of their diameter and chirality distributions. In Fig. 4(a), the diameter distributions are displayed, which were obtained by Raman measurements using three different excitation wavelengths (488, 514.5, and $633 \mathrm{~nm})$. Nanotube diameter was estimated from the position of the radial breathing mode (RBM) using the relationship $d=248 / \omega_{\mathrm{RBM}},{ }^{(9)}$ where $d$ and $\omega_{\mathrm{RBM}}$ are the diameter 


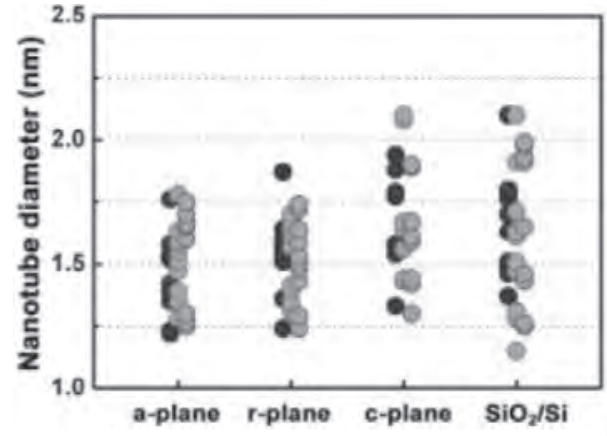

(a)

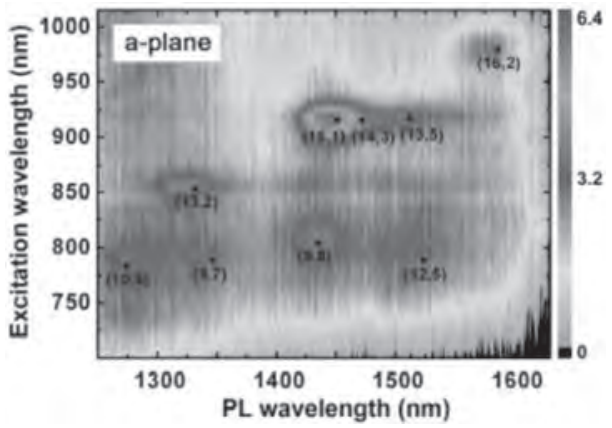

(c)

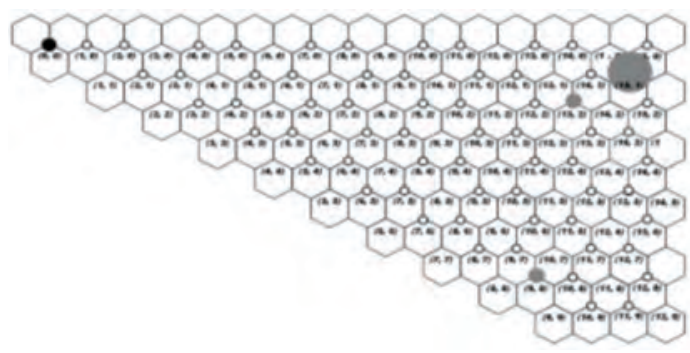

(e)

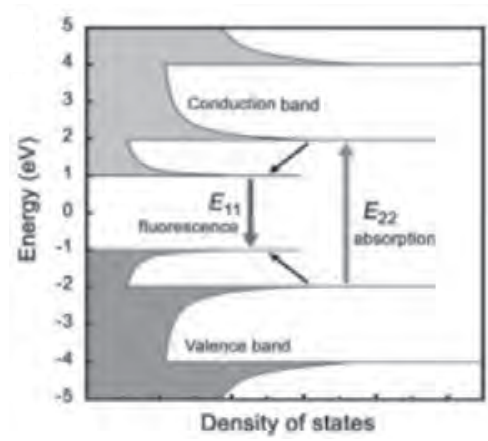

(b)

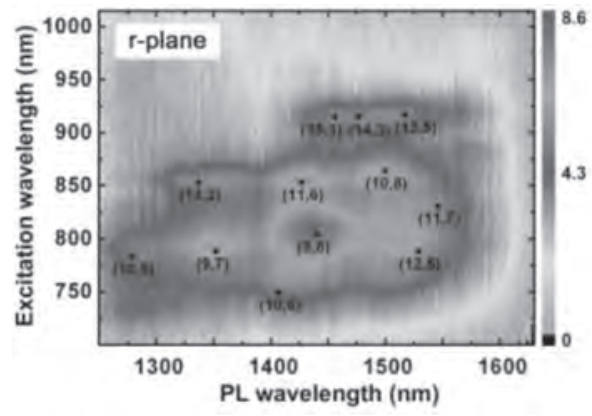

(d)

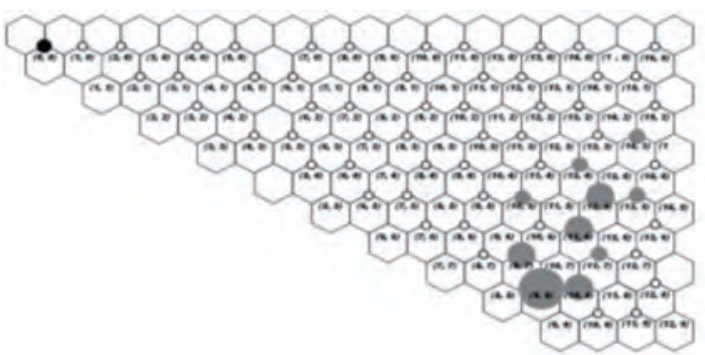

(f)

Fig. 4. (a) Diameter distribution determined by Raman spectra measurements using three different wavelengths $(488,514.5$, and $633 \mathrm{~nm})$. The SWNTs grown on four different substrates are compared. (b) Typical density of states of semiconducting SWNT. PL contour maps of aligned SWNTs on a- (c) and r-planes (d). (e,f) Relative PL intensity plotted on chiral map. 
and RBM frequency, respectively. For comparison, the distributions of randomly oriented SWNTs grown on the c-plane sapphire and $\mathrm{SiO}_{2} / \mathrm{Si}$ substrates are also plotted. We found that the aligned SWNTs grown on sapphire $r$ - and a-planes have smaller diameters and narrower distributions compared with the randomly oriented SWNTs on c-plane sapphire. This can be explained by the surface energy of each surface: the $r$ and a-planes have higher surface energies than the c-plane so that small Co-Mo metal nanoparticles were stabilized on the $\mathrm{r}$ - and a-planes at the high reaction temperature of $900^{\circ} \mathrm{C}$.

Near-infrared photoluminescence (NIR-PL) spectroscopy is a powerful means to study the chirality distribution of SWNTs. ${ }^{(10)}$ The combination of $E_{11}$ and $E_{22}$ transition energies between sharp branches of the valence and conduction bands depends on nanotube chirality (Fig. 4(b)). By measuring a PL spectrum with changes in excitation wavelength, one can obtain information on the chirality distribution of semiconducting SWNTs. PL contour maps were obtained for the aligned SWNTs on the a- and r-plane sapphires, as shown in Figs. 4(c) and 4(d), respectively.(11) The observed PL was broad and very weak, because the SWNTs were attached to the substrate. The observed PL intensities are plotted in chiral maps (Figs. 4(e) and 4(f)). In spite of the fact that the two samples were prepared under the same CVD condition $\left(800^{\circ} \mathrm{C}\right)$ using the same catalyst (Co-Mo), these two samples showed very different PL features. On the a-plane, the PL from the near zigzag structure, mainly from $(15,1)$, was strong. On the other hand, the SWNTs on the r-plane showed strong PL from near armchair structures with strong emissions from $(9,8),(9,7),(10,8),(11,6)$, and $(12,5)$ nanotubes. It is widely known that the as-synthesized SWNTs show strong PL from near armchair structures. $^{(10)}$ Therefore, the PL observed for the aligned SWNTs on the a-plane (Figs. 4(c) and 4(e)) is very interesting. Our results suggest that not only the growth direction but also the nanotube structure was modified by the sapphire substrate. However, as the photodetector used for this study cannot detect the PL from SWNTs whose diameters are larger than $1.4 \mathrm{~nm}$, we plan to synthesize aligned SWNTs with smaller diameters for further control of the SWNT structure on the basis of our "epitaxial nanotube growth" concept.

Assuming the base-growth mode for the aligned SWNTs, the chirality of one SWNT is supposed to be basically the same along its entire length. In this mode, the chirality of the nanotube is determined at the early stage of its growth, that is, the nucleation step of the SWNT. Hence, we consider that an understanding of the relationship among the substrate-catalyst-cap is essential for the highly controlled SWNT growth.

\section{Aligned Growth on Si Wafers}

The horizontally aligned growth on a substrate enables the efficient fabrication and improved performance of SWNT-based devices. A group at the University of Illinois demonstrated the fabrication of a radio that was operated using an aligned SWNT array grown on a quartz substrate. ${ }^{(12)}$ Because the sapphire and quartz substrates are rigid and good insulators, the techniques of transferring aligned SWNTs to polymers (for flexible 
electronics) and Si substrates have been intensively studied.(13,14) For high-performance applications, such as large-scale integration devices (LSI), it is required to assemble SWNTs on a Si wafer with an oxidized layer $\left(\mathrm{SiO}_{2} / \mathrm{Si}\right)$. However, the transfer of aligned SWNTs from sapphire or quartz has several drawbacks, such as cost and the introduction of defects and contamination during the transfer.

Recently, we have observed that SWNTs are aligned radially on the Si wafer treated with $\mathrm{CF}_{4}$ plasma. ${ }^{(15)}$ The plasma treatment formed radial trenches on the $\mathrm{SiO}_{2}$ surface with a depth of $\sim 5 \mathrm{~nm}$. These trenches guided the SWNT growth, giving high-density

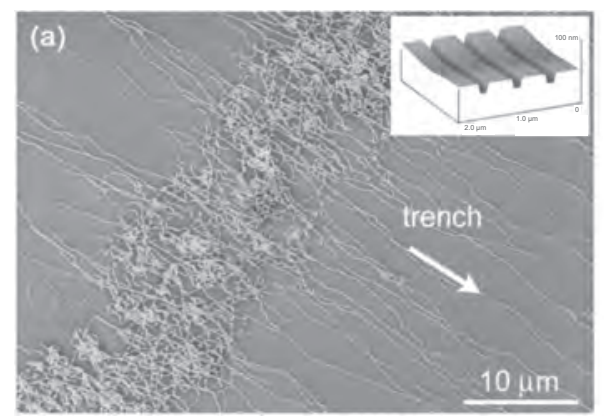

(b)
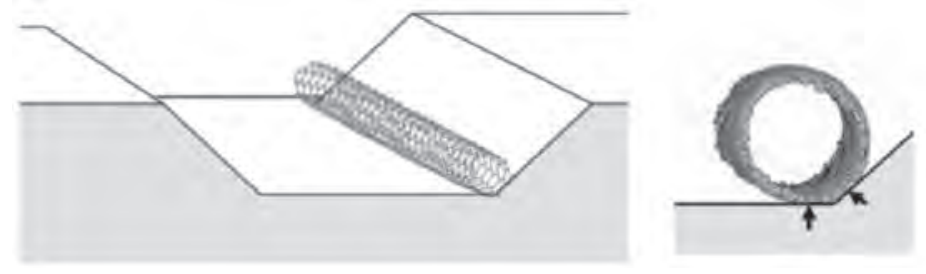

(c)

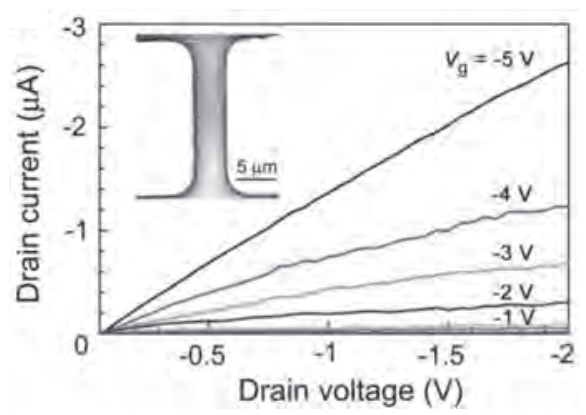

Fig. 5. (a) SEM image of aligned SWNTs grown on trenched Si wafer. Inset: AFM image of trench structure. (b) Schematic illustration of SWNT aligned along trench edge. (c) FET characteristics measured for aligned SWNT array. Data were collected after electrical breakdown. 
aligned SWNT arrays that became radically arranged.

We exploited this finding by forming artificial trench structures on the basis of the top-down approach. We formed periodic trenches by electron beam (EB) lithography and reactive ion etching (RIE), and succeeded in producing aligned SWNTs along these trenches, as shown in Fig. 5(a). ${ }^{(16)}$ The SWNTs were aligned along the trench direction, and it is considered that the SWNT is attached at the corner of the trench. This position is stabilized by the van der Waals interactions from both the side wall and the bottom of the trench (Fig. 5(b)). However, the degree of alignment is not as high as that on single crystals. We infer that this originated from the nanoscale roughness of the artificial trenches and can be improved by optimizing the process conditions. Using a $\mathrm{Si}$ wafer $\left(\mathrm{SiO}_{2} /\right.$ Si), we fabricated a bottom-gate FET with aligned SWNTs (Fig. 5(c)). The breakdown of metallic SWNTs by passing high current gave a good transistor performance with an on-off ratio of $10^{3}$ and a mobility of $5 \mathrm{~cm}^{2} / \mathrm{V} \cdot \mathrm{s}$. This mobility is much higher than that of typical organic transistors $\left(0.1 \mathrm{~cm}^{2} / \mathrm{V} \cdot \mathrm{s}\right) \cdot{ }^{(17)}$

\section{Conclusions}

We present our recent findings on horizontally aligned SWNT growth and the growth mechanism. The aligned growth will enable the integration of a number of SWNTs through the control of growth direction and position, and is expected to lead to development of electronic applications. Furthermore, our finding of the aligned growth on the surface-modified Si wafers opens up the possibility of integrating SWNTs into modern Si electronics. The controlled growth of SWNTs is highly desired, and the present epitaxial interaction might provide a new route to realizing full control.

\section{Acknowledgements}

We thank Professor K. Takahashi and K. Ikuta for help in lithography processes, Dr. K. Ikeda for helpful discussion, and Dr. N. Minami and Dr. K. Iakoubovskii for PL measurements. This work was supported by PRESTO-JST, Nanoelectronics project of NEDO, a Grant-in-aid for Scientific Research from MEXT, and Industrial Technology Research Program from NEDO, Japan.

\section{References}

1 A. Javey and J. Kong (ed.): Carbon Nanotube Electronics (Springer-Verlag, Berlin, 2009).

2 H. Ago, K. Nakamura, K. Ikeda, N. Uehara, N. Ishigami and M. Tsuji: Chem. Phys. Lett. 408 (2005) 433.

3 H. Ago, N. Uehara, K. Ikeda, R. Ohdo, K. Nakamura and M. Tsuji: Chem. Phys. Lett. 421 (2006) 399.

4 S. Han, X. Liu and C. Zhou: J. Am. Chem. Soc. 127 (2005) 5294.

5 C. Kocabas, S. H. Hur, A. Gaur, M. A. Meitl, M. Shim and J. A. Rogers: Small 1 (2005) 1110.

6 N. Ishigami, H. Ago, T. Nishi, K. Ikeda, M. Tsuji, T. Ikuta and K. Takahashi: J. Am. Chem. Soc. 130 (2008) 17264. 
7 Y. Miyauchi and S. Maruyama: Phys. Rev. B 74 (2006) 35415.

8 H. Ago, N. Ishigami, N. Yoshihara, K. Imamoto, S. Akita, K. Ikeda, M. Tsuji, T. Ikuta and K. Takahashi: J. Phys. Chem. C 112 (2008) 1735.

9 A. M. Rao, E. Richter, S. Bandow, B. Chase, P. C. Eklund, K. A. Williams, S. Fang, K. R. Subbaswamy, M. Menon, A. Thess, R. E. Smalley, G. Dresselhaus and M. S. Dresselhaus: Science 275 (1997) 187.

10 S. M. Bachilo, M. S. Strano, C. Kittrell, R. H. Hauge, R. E. Smalley and R. B. Weisman: Science 298 (2002) 2361.

11 N. Ishigami, H. Ago, K. Imamoto, M. Tsuji, K. Iakoubovskii and N. Minami: J. Am. Chem. Soc. 130 (2008) 9918.

12 C. Kocabas, H. Kim, T. Banks, J. A. Rogers, A. A. Pesetski, J. E. Baumgardner, S. V. Krishnaswamy and H. Zhang: Proc. Nat. Acad. Sci. 105 (2008) 1405.

13 S. J. Kang, C. Kocabas, T. Ozel, M. Shim, N. Pimparkar, M. A. Alam, S. V. Rotkin and J. A. Rogers: Nat. Nanotechnol. 2 (2007) 230.

14 L. Jiao, B. Fan, X. Xian, Z. Wu, J. Zhang and Z. Liu: J. Am. Chem. Soc. 130 (2008) 12612.

15 N. Yoshihara, H. Ago, K. Imamoto, M. Tsuji, T. Ikuta and K. Takahashi: J. Phys. Chem. C 113 (2009) 8030.

16 C. M. Orofeo, H. Ago, N. Yoshihara and M. Tsuji: Appl. Phys. Lett. 94 (2009) 53113.

17 A. Dodabalapur: Mater. Today 9 (2006) 24. 